\title{
EDITORIAL
}

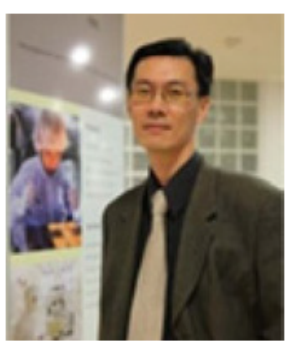

\section{Special Issue on Biomechanics}

\author{
Han Sung Kim \\ (Co-guest Editor on Special Issue)
}

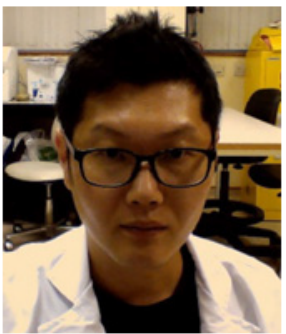

\section{Seungkwan Cho}

(Co-guest Editor on Special Issue)

(C) The Korean Society of Medical \& Biological Engineering and Springer 2015

Biomechanics is the study of the structure and function of biological systems as humans, animals, and plants by means of the methods of mechanics. The field of biomechanics ranges from the inner workings of a cell to the development and movement of living things. Thus, biomechanics considers as an emerging multidisciplinary engineering that is closely related to the general mechanics, cell/tissue biology, and medicine. Some general examples of biomechanics research include the investigation of the forces that act on human body, the aerodynamics of bird or insect flight, the hydrodynamics of swimming in fish, and locomotion in general across all forms of life, from individual cells to whole organisms.

The traditional biomechanics of human beings is a core part of kinesiology. As we develop a greater understanding of the physiological behavior of living tissues, researchers are able to advance the field of tissue engineering, as well as develop improved treatments for a wide array of pathologies. Biomechanics is also adopted to studying human musculoskeletal systems. Such research utilizes force platform to study human

Han Sung Kim $(\bowtie)$

Department of Biomedical Engineering, College of Health Science, Yonsei University, Wonju, Gangwon 220-710, Korea

Tel : +82-33-760-2913/Fax : +82-33-760-2912

E-mail : hanskim@yonsei.ac.kr

Seungkwan Cho( $(\mathrm{Q})$

Yonsei-Fraunhofer Medical Device Lab., Wonju, Gangwon, Korea

Tel : +82-33-760-2913/ Fax : +82-33-760-2912

E-mail : biecsk@naver.com ground reaction force and infrared videography which is captured by trajectories of reflective markers to study human 3D motion. This research applies electromyography (EMG) system to study the muscle activation as well. By this, it is feasible to investigate the muscle responses to the external forces as well as perturbations. In addition, biomechanics is widely used in orthopedic industry to design orthopedic implants for human joints, dental parts, external fixations and other medical purposes. In here, bio-tribology is a most important part of it. It is a study of the performance and function of biomaterials used for orthopedic implants. It plays a vital role to improve the design and produce successful biomaterials for medical and clinical purposes.

Meanwhile, from an engineering point of view, the application of principle to human cannot fully reveal the actual phenomena. Some simple applications of general mechanics and material sciences can supply correct approximations to the mechanics of many biological systems. However, actual biological systems are much more complex than man-built systems. Numerical analysis techniques are hence utilized in various biomechanical studies as an altemative way to estimate the various phenomena under various conditions and situations. Numerical technique is performed by an iterative process of hypothesis and verification, including several steps of modeling, computational simulation, and experimental measurements.

In here, we can summarize above mentioned contents that the biomechanics is widely applied in the field of sports, 
material sciences, assistive technologies and medicine, and which can be performed by experiments and computational simulation. From a clinical point of view, due to its importance in human health, we select biomechanics as a theme of this Special Issue of Biomedical Engineering Letters. This Special Issue introduces five original articles covering various stateof-the-art technologies, which include bone mechanics, motion analysis, rehabilitation engineering and assistive technology.

The first introducing paper entitled "Enhancement of bone quality and longitudinal growth due to free-fall motion in growing rat" is reported by Cho and co-authors. Many people are interest in the effects of exercise whether it can be helpful to prevent aging or to accelerate growth. This study tries to elucidate the relation between the physical exercise and the enhancement of bone strength and longitudinal growth. Based on the results, they reported that high impact loading exercise such as landing motion may enhance the bone quality and longitudinal length at the same time. This study can provide a clue that the physiological changes can be effective by human activity. This kind of studies are classified as biomechanics.

The second paper entitled "Effect of stool height on slumped posture during push-and-pull weeding motion" is trying to suggest an appropriate working posture to prevent the musculoskeletal disorders or pain. The authors particularly focused on the working posture for farm working. This study analyzed the lower body joint torques and muscle contractions for various stool heights with a slumped posture during push-and-pull weeding. In this study, 3D motion analysis and dynamic simulation techniques were used. This study seems to be a proper example to show the combined techniques between 3D motion analyses by tracking marker and dynamic simulation by using computational model.

The third and fourth papers entitled "Suggestion of new concept for mobility assistive system based on wheelchair platform with gait assistive function controlled by artificial pneumatic muscle" and "Characteristics of center of body mass trajectory and lower extremity joint motion responded by dynamic motions of balance training system" are commonly described for application of rehabilitation and assistive system with respect to elderly or disabled people. These kinds of studies are significant not only human welfare but also new formation of industry. Even though computational simulation cannot substitute for entire experiment, it can consider effective way to supplement with sophisticated results.

The fifth paper entitled "Lower body motion analysis to detect falls and near falls on stairs" is referred to assistive technology that the authors have developed a methodology for computer vision system to automatically detect abnormal events during stair traversal and experimentally validated it on a ground truth dataset collected in a smart home environment. In the actual situation, older adults are especially exposed to dangerous situations on stairways because of their weakened visual and musculoskeletal systems. The ability to automatically detect and predict falls can be helpful to provide prompt assistance when an accident does occur. This kind of study is very useful to prevent risk of injuries such as bone fractures or damage to the muscles and internal organs. Biomechanics can deal with it up to realistic application for prevention of risk and assistance of daily life.

We briefly introduced the application of biomechanics in here, but the application of biomechanics has a much wider range than above mentioned contents. We would like to express our gratitude to the authors who present through overview of various state-of-the-art technologies and highlight their application of biomechanics. We would also like to appreciate to the Managing Editor (Prof. Jae Sung Lee) and Editor-in-Chief (Prof. Sang-Hoon Lee) of the Biomedical Engineering Letters for their support and contribution of this Special Issue.

\section{REFERENCES}

[1] Cho SK, Eom S, Seo D-H, Park J, Ko C-Y, Kim HS. Enhancement of bone quality and longitudinal growth due to free-fall motion in growing rats. Biomed Eng Lett. 2015; 5(2):73-8.

[2] Cho YK, Myeong S, Cho S, Kim HS. Effect of stool height on slumped posture during push-and-pull weeding motion. Biomed Eng Lett. 2015; 5(2):79-86.

[3] Lee B, Ko C, Ko J, Kim JS, Lim D. Suggestion of new concept for mobility assistive system based on wheelchair platform with gait assistive function controlled by artificial pneumatic muscle. Biomed Eng Lett. 2015; 5(2):87-91.

[4] Jung H, Han J, Kim CY, Chun KJ, Jung D, Kim JS, Lim D. Characteristics of center of body mass trajectory and lower extremity joint motion responded by dynamic motions of balance training system. Biomed Eng Lett. 2015; 5(2):92-7.

[5] Parra-Dominguez GS, Snoek J, Taati B, Mihailidis A. Lower body motion analysis to detect falls and near falls on stairs. Biomed Eng Lett. 2015; 5(2):98-108. 